\title{
Influence of Interphase Properties on the Behavior of Wavy Carbon Nanotube Reinforced Composites
}

\author{
Emre Köroğlu, Halit S. Türkmen \\ Istanbul Technical University \\ Faculty of Aeronautics and Astronautics, Maslak, Istanbul, Turkey \\ yuzbasiog2@itu.edu.tr; halit@itu.edu.tr
}

\begin{abstract}
CNTs (carbon nanotubes) are ideal reinforcing materials for high strength polymer nanocomposites because of their high stiffness, strength and resilience properties. CNT-matrix interface is one of the parameters effecting the stiffness of nanocomposites. In this study, the effect of CNT-matrix interface properties on the effective global stiffness of nanocomposites is investigated numerically. A representative volume element (RVE) is modeled by using the finite element method (FEM) to predict the elastic behavior in micro level. The finite element model consists of three phases namely matrix, CNT and interphase. The stiffness is obtained from the elastic response of RVE subjected to an axial loading. The stiffness values are obtained for several thickness and modulus values of the interface and the waviness of CNT. Effective global stiffness is calculated by using Mori-Tanaka method where CNTs have varying amount of waviness, interphase properties and random orientations. It is found that there are significant effects of the interface on the effective global stiffness.
\end{abstract}

Keywords: Carbon nanotube, waviness, stiffness, composite, Mori-Tanaka, interphase

\section{Introduction}

Superior mechanical, electrical and thermal properties of carbon nanotubes (CNTs) resulted in a boost of research activity since their discovery in 1991 [1]. Basically CNTs are tubular structures composed of hexagonal meshes of carbon atoms at nanoscale with high stiffness and tensile strength properties. Considering the fact that their density is half of aluminum and their strength is twenty times greater than high strength steel alloys [2], even with a small volumetric contribution in a material, CNTs can increase the stiffness and strength by a considerable amount. On the other hand CNTs can be used in favor of crashworthiness by means of their nanotube breakage mechanism [3]. CNTs can be used in many industrial applications. The Boeing Company had already granted a patent for a material with CNTs [4]. Superior crashworthiness properties make CNT reinforced composites a good candidate for future generation of automotive materials. Mechanical aspects of CNTs have been popular topics of interest in the literature. Due to the curvature of their profile, waviness of CNTs have detrimental effects on axial stiffness [5,6]. The interaction between CNT inclusions and the matrix material is another important issue to consider while modeling reinforced composites. A review of recent work on interphase engineering and modeling can be found in Karger-Kocsis et al [7].

To simulate the CNT reinforced composites, there are many tools available with different levels. For example Liu and Chen used finite element method to model a single CNT inside a representative volume of matrix [8]. Li and Chou constructed a molecular level FEM analysis with hexagonal mesh structure using beam elements where the stiffness of the beams have been used as analogies for force fields between atoms [9]. CNTs have also been modeled directly at molecular level by using molecular dynamics [10]. It is very difficult to obtain global characteristics by using molecular dynamics or FEM directly. Therefore an approximate homogenization approach is preferred. Seidel and Lagoudas used Mori-Tanaka method to obtain effective axial modulus of CNT reinforced composites [11].

In this study, both interfacial and waviness effects have been considered. First, an RVE model has been constructed for micro level CNT elastic behavior. Then based on those results, effective global stiffness has been calculated by MoriTanaka method where CNTs have varying amount of waviness, interphase properties and random orientations. 


\section{Modeling}

From a mechanical point of view, elastic coefficients of a carbon nanotube reinforced composite is of primary interest. Considering the number of CNTs in the matrix material along with their wavy geometry and random orientations, it would be infeasible to use finite element method directly. For that reason, a two level approach has been utilized in this study. At the first level, a representative volume element (RVE) is constructed. This model can represent the matrix-CNT collective behavior at micro scale. The main information extracted at this level is the impact of local properties such as waviness and interphase. At the second level, the extracted information about single carbon nanotube along with global properties like volume fraction, orientation and probabilistic distribution of local properties, are used in a homogenization procedure based on the work of Mori and Tanaka [12]. In the following subsections, methods and the corresponding parameters are presented in detail.

\subsection{RVE Model}

An RVE is versatile model to gather information from a micro-structure. The model consists of two main phases of materials representing the matrix and the carbon nanotube with a third additional interphase to take into account the effect of the interaction between the CNT and the matrix material. The geometry and mesh structure of the model is shown in Figure 1. A prismatic shape is selected for the bulk matrix and a generic wavy profile is constructed that is defined by the following expression:

$$
\operatorname{Asin}\left(\frac{2 \pi z}{\lambda}\right)
$$

where $\mathrm{A}$ is the amplitude, $\lambda$ is the wavelength of the wavy curve $\mathrm{z}$ is the distance from the rear face of the RVE where CNT curve ends. Using quarter symmetry, the model has symmetric boundary conditions on $\mathrm{x}=0$ and $\mathrm{z}=\lambda / 2$. The finite element model is constructed using 3D solid elements for the matrix, and layered shell elements for the CNT and the interphase due to its tubular shape. Isotropic material properties have been chosen for all three phases.

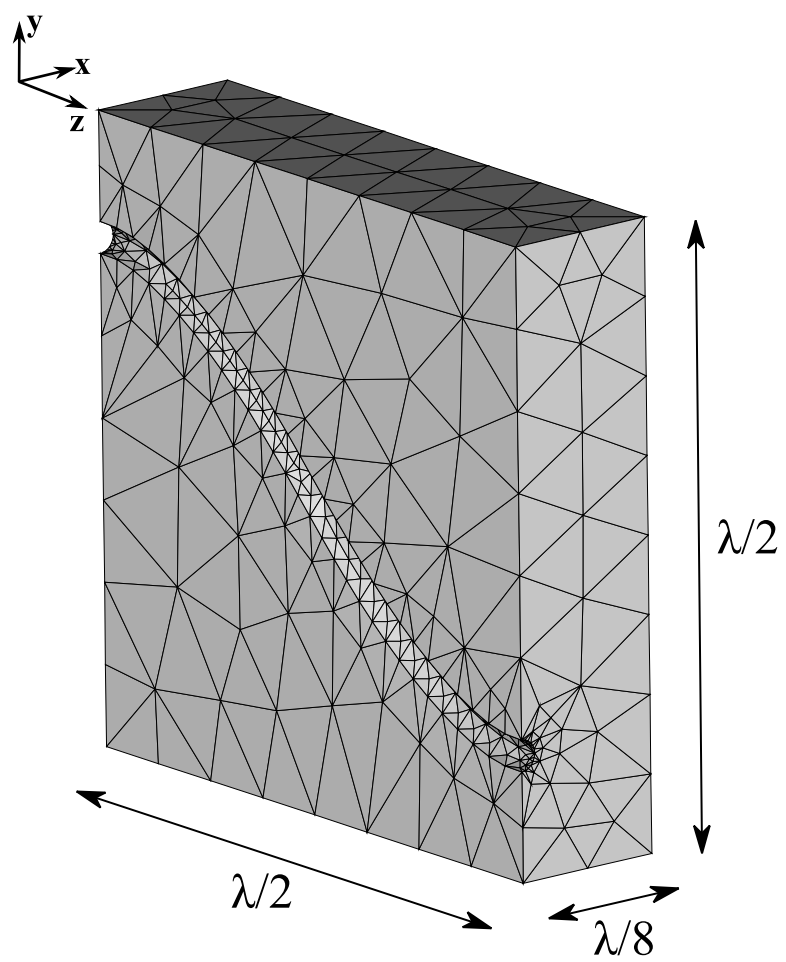

Fig. 1: RVE model. 
To determine the effective reinforcing moduli [5], the model is subjected to a small amount of axial displacement $\delta$ in axial (z) direction. Using the reaction force $F$, which is calculated by finite element analysis (FEA) and the Area of the cross-section (rear face parallel to $\mathrm{x}-\mathrm{y}$ plane), the effective elastic modulus of RVE $\left(E_{R V E}\right)$ is obtained by following equation:

$$
E_{R V E}=\frac{F\left(\frac{\lambda}{2}\right)}{\text { Area. } \delta}
$$

Finally using rule of mixtures, the equivalent reinforcing moduli of the CNT is obtained as,

$$
E_{e q v}=\frac{E_{R V E}-\left(1-v f_{C N T}\right) \cdot E_{M}}{v f_{C N T}}
$$

where $v f_{c n t}$ is the volume fraction of CNT and $E_{M}$ is the modulus of elasticity of matrix. To calculate the volume fraction, volume of the CNT is calculated by using the following equation:

$$
\int_{0}^{\lambda / 2} \sqrt{1+\left(\frac{d y}{d z}\right)^{2}} d z \times A_{c s}
$$

where $A_{c s}$ is the cross sectional area of CNT. To have a parametric version of the volume expression, the integral is evaluated using seven point Simpson integral approximation. Finally approximate expression for the volume is obtained as,

$$
\text { volume }(A, \lambda)=\frac{\lambda}{36}\left(2+8 \sqrt{1+\frac{A^{2} \pi^{2}}{\lambda^{2}}}+4 \sqrt{1+\frac{3 A^{2} \pi^{2}}{\lambda^{2}}}+4 \sqrt{1+\frac{4 A^{2} \pi^{2}}{\lambda^{2}}}\right) x A_{c S}
$$

\subsection{Mori-Tanaka Homogenization}

After calculating the axial elastic modulus of the RVE, an effective elasticity matrix on a global sense is determined. It is considered that there are many CNTs distributed inside the material with different waviness, interphase properties, orientations, etc. Therefore some form of homogenization for the inclusions must take place to have a global representative material information.

Eshelby [13], introduced a method to calculate effective material properties of an infinite matrix with an ellipsoid inclusion. This method assumed that inclusions are dilute i.e. they are non-interacting or otherwise the interactions are weak. Mori and Tanaka [12] method based on Eshelby's work, can model inclusions with higher volume fractions with interactions. The effective stiffness matrix due to Mori-Tanaka is given by

$$
\boldsymbol{C}^{*}=\boldsymbol{C}_{\mathbf{1}}+c 2\left(\boldsymbol{C}_{\mathbf{2}}-\boldsymbol{C}_{\mathbf{1}}\right) \boldsymbol{T}(c 1 \boldsymbol{I}+c 2 \boldsymbol{T})^{-1}
$$

where $\boldsymbol{C}_{\mathbf{1}}$ and $\boldsymbol{C}_{\mathbf{2}}$ are the stiffness tensors of matrix and inclusions respectively, $\boldsymbol{I}$ is the identity tensor and $\boldsymbol{T}$ is the concentration tensor given by

$$
T=\left[I-S C_{1}^{-1}\left(C_{1}-C_{2}\right)\right]^{-1}
$$

where $\boldsymbol{S}$ is the Eshelby tensor depending on the geometry of the inclusion and $\boldsymbol{C}_{2}$ is the orientational and volumetric average stiffness matrix of the multiple inclusions in both equations. 
The CNTs have varying waviness and orientations. Therefore those variations are calculated in RVE phase and included in the Mori-Tanaka calculations by a probabilistic distribution in terms of volume fractions.

To obtain a general idea of the axial stiffness of the reinforced material, reciprocal of the first diagonal term of the anisotropic compliance tensor is used as representative elastic modulus. So global effective stiffness term is obtained as,

$$
E_{e f f}=\frac{1}{S_{11}}
$$

where $S$ is the anisotropic compliance tensor from Mori-Tanaka calculation.

\section{Results}

Depending on the interaction between the matrix material and CNT, the overall elastic behavior may differ. This study focuses on the effects of the interphase properties, especially the interface wall thickness and the modulus of elasticity.

Parametric analyses for both RVE and Mori-Tanaka method have been achieved. The parameter values are listed in Table 1 for RVE and Table 2 for Mori Tanaka method. The values inside curly brackets are sample points for parametric studies.

Table 1: RVE parameters.

\begin{tabular}{|c|c|}
\hline Wavelength of CNT curve $(\lambda)[\mu \mathrm{m}]$ & 0.4 \\
\hline CNT Length $\left(L_{R V E}\right)[\mu \mathrm{m}]$ & 0.2 \\
\hline CNT Width $\left(W_{R V E}\right)[\mu \mathrm{m}]$ & 0.05 \\
\hline CNT Height $\left(H_{R V E}\right)[\mu \mathrm{m}]$ & 0.2 \\
\hline Poisson ratio for all three phases $(v)$ & 0.3 \\
\hline Modulus of elasticity of matrix $\left(E_{M}\right)[\mathrm{GPa}]$ & 1.0 \\
\hline Modulus of elasticity of CNT $\left(E_{C N T}\right)[\mathrm{GPa}]$ & $\{50,100,200,400\}$ \\
\hline Modulus of elasticity of interphase $\left(E_{I N T}\right)[\mathrm{GPa}]$ & $\{10,20,50,100\}$ \\
\hline CNT diameter $\left(d_{C N T}\right)[\mathrm{nm}]$ & $\{0.6,0.9,1.2,2.0\}$ \\
\hline Thickness ratio of interphase and CNT $\left(t p_{I N T}\right)$ & $\{0.2,0.6,1.0,1.5\}$ \\
\hline Amplitude of CNT curve $(A)[\mu \mathrm{m}]$ & $\{0.00,0.02,0.04,0.08,0.12\}$ \\
\hline
\end{tabular}

Table 2: Mori-Tanaka parameters.

\begin{tabular}{|c|c|}
\hline Number of waviness types $\left(n_{\text {inc }}\right)$ & 5 \\
\hline Number of repetitions for each inclusion $\left(r e p_{\text {inc }}\right)$ & 40 \\
\hline Amplitude of CNT curve $(A)[\mu \mathrm{m}]$ & $\{0.00,0.02,0.04,0.08,0.12\}$ \\
\hline Probability mass function of each inclusion type $\left(p_{\text {wavy }}\right)$ & $\{0.05,0.15,0.30,0.30,0.20\}$ \\
\hline Volume fractions of CNT inclusions $\left(v f_{\text {cnt }}\right)$ & $\{0.02,0.05,0.1,0.2\}$ \\
\hline
\end{tabular}

For parametric analyses, some of the parameters have been kept constant at their nominal values which are listed in Table 3.

Table 3: Nominal values of parameters.

\begin{tabular}{|c|c|}
\hline$E_{C N T}[\mathrm{GPa}]$ & 200 \\
\hline$E_{I N T}[\mathrm{GPa}]$ & 50 \\
\hline$d_{C N T}[\mathrm{~nm}]$ & 1 \\
\hline$t p_{I N T}$ & 0.75 \\
\hline$A[\mu \mathrm{m}]$ & 0.08 \\
\hline
\end{tabular}


Four of the six case studies shown in Fig.2-Fig.7 deal with RVE. In Fig.2 the effect of waviness (represented by the amplitude of CNT wavy curve) and modulus of elasticity of CNT is shown. It is clear that increase in waviness and decrease in $E_{C N T}$ results in lower $E_{\text {eqv }}$. Besides high waviness have more detrimental effect on higher values of $E_{C N T}$. In Fig.3, the effect of interphase elasticity could be seen. Although there is a tenfold decrease in $E_{I N T}$, the corresponding change in outcome is not as high. Like $E_{C N T}$, when $E_{I N T}$ decreases $E_{\text {eqv }}$ decreases as well. When the diameter of the CNT increases (Fig.4), the resistance to shrink transversely decreases and in turn it causes the axial elastic modulus to decrease. On the other hand, waviness has a higher impact for smaller diameters. As $t p_{I N T}$ decreases, the share of the CNT increases and as a result the effective elastic modulus increases (Fig.5). Increasing the $E_{I N T}$ also increases $E_{\text {eqv }}$, but when the fraction of interphase is higher the increase of $E_{\text {eqv }}$ is faster.

In the second phase, the effect of CNT volume fraction on effective global modulus is investigated. As can be seen in Fig.6, increasing the $E_{I N T}$ results in a substantial increase of $E_{\text {global }}$ when the volume fraction of CNT is high. Finally in Fig.6, lower fractions of interphase mean more effective CNT therefore global effective modulus have a proportional gain. The resulting trends are not uniform. This is mainly due to the fact that limited number of inclusion types $\left(\right.$ rep $\left._{\text {inc }}\right)$ has been used to get a global estimate.

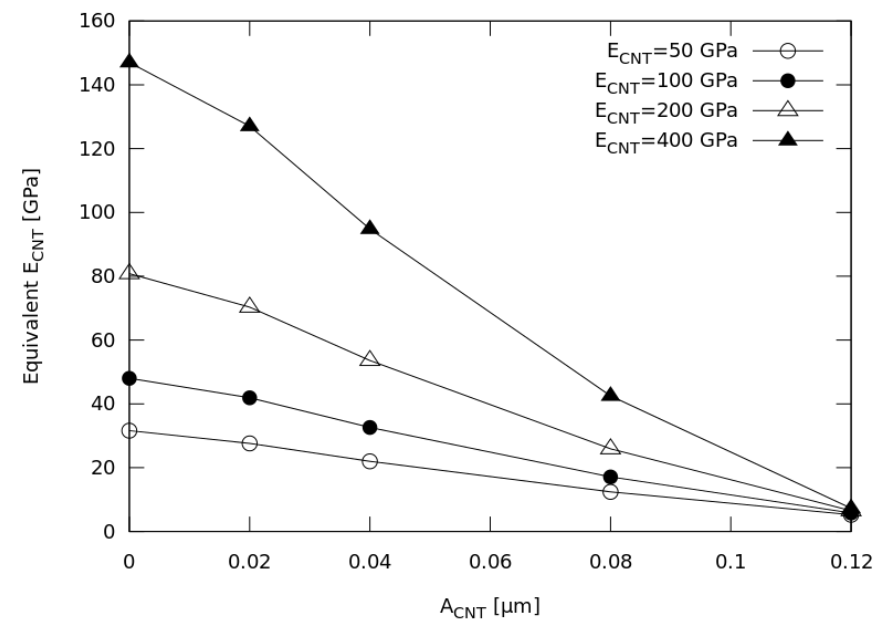

Fig. 2: $E_{C N T}$ effect on $E_{\text {eqv }}$.

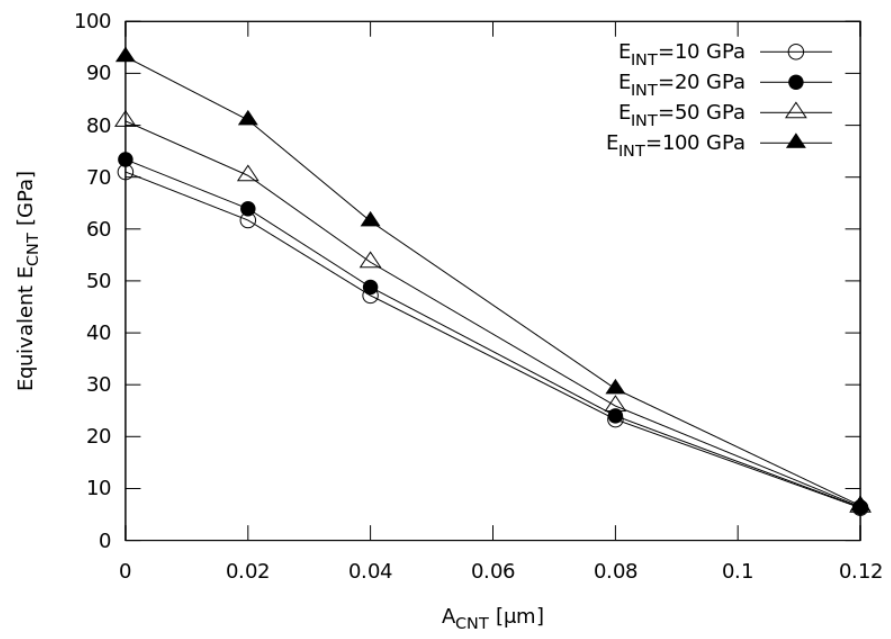

Fig. 3: $E_{I N T}$ effect on $E_{e q v}$. 


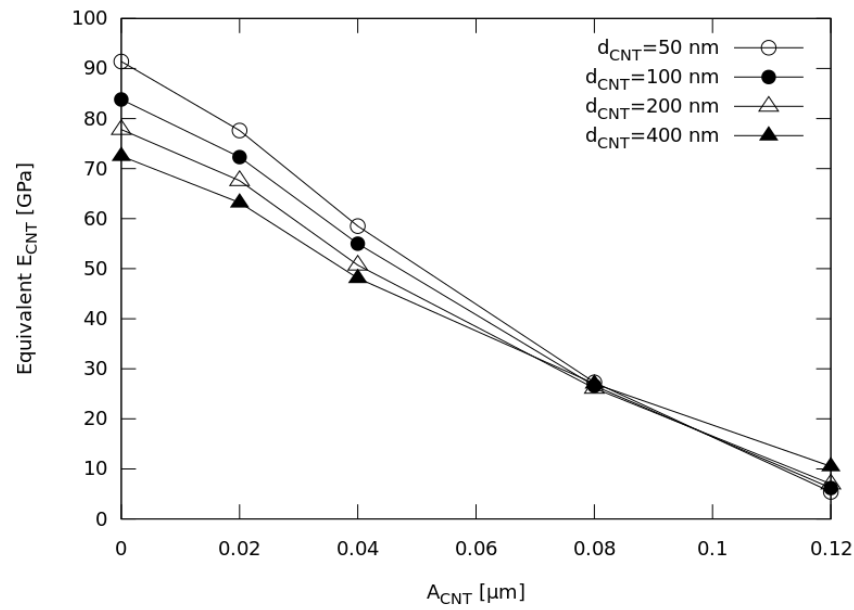

Fig. 4: $d_{C N T}$ effect on $E_{e q v}$.

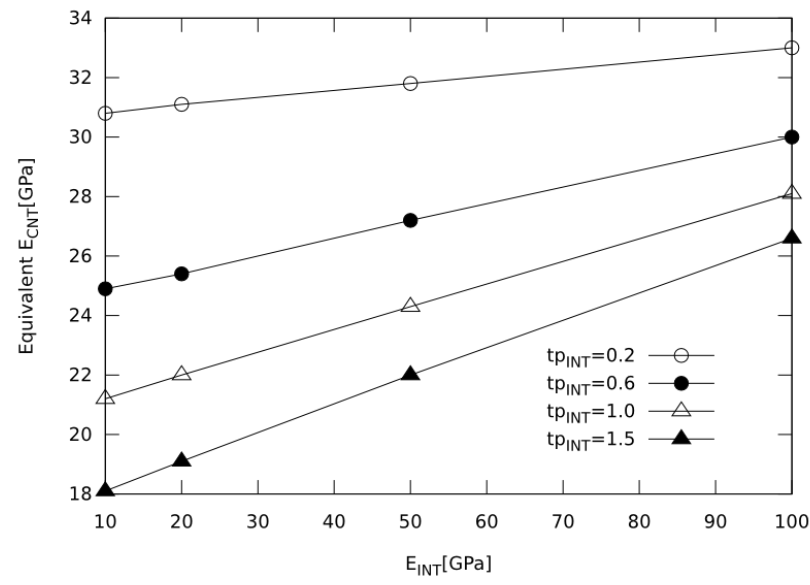

Fig. 5: $t p_{I N T}$ effect on $E_{e q v}$.

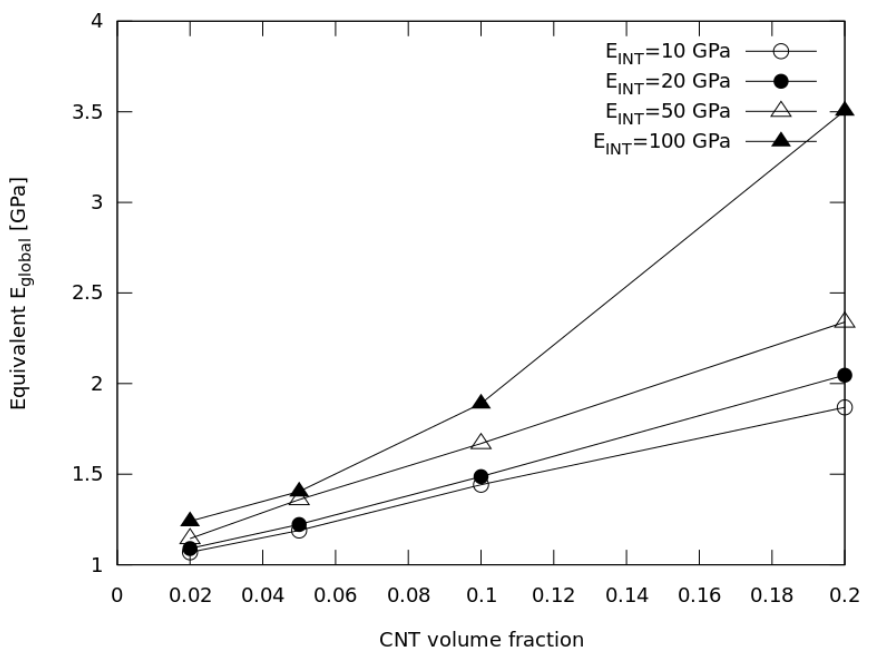

Fig. 6: $E_{C N T}$ effect on $E_{\text {global }}$. 


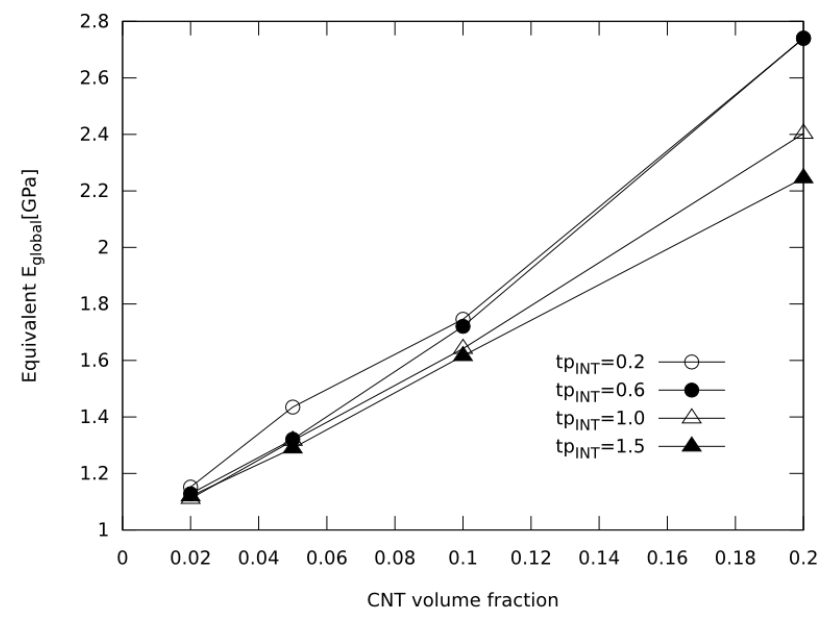

Fig. 7: $t p_{I N T}$ effect on $E_{\text {global }}$.

\section{Conclusion}

Main aim of this study is to quantify the effect of interfacial relations between matrix and CNT on the global effective axial modulus. Due to high computational requirements of direct FEA of CNT reinforced composites, a two level approach has been followed. At the first level, an RVE model is constructed and analyzed using FEA. Interphase properties have a significant impact on effective modulus of CNT, but not as much as the properties of CNT itself. At the second level, a homogenization method is utilized. At the global level, the interphase again has a considerable role on elastic modulus. Due to probabilistic distribution of elastic properties of CNT inclusions, the results were somehow not as regular as the local counterparts.

The approach in this paper could be used for practical applications. For instance, composite simulation software can incorporate the effect of interphase and waviness of CNTs at nano-scale RVE models. Another example could be the prediction of moduli for CNT reinforced epoxies used for the construction of sandwich panels to increase the interface strength between layers. Besides the user may need a further error analysis due to the fact that no consensus exists for both the number of samples of inclusion types and their corresponding probability mass function. A reliability based design optimization study would benefit from the calculated uncertainties.

\section{Acknowledgement}

This research was supported by Council of Higher Education of Turkey under the OYP program.

\section{References}

[1] S. Iijima, "Helical microtubules of graphitic carbon," Nature, vol. 354, no. 6348, pp. 56-58, 1991.

[2] P. G. Collins, P. Avouris, "Nanotubes for electronics," Scientific American, vol. 283, no. 6, pp. 62-69, 2000.

[3] H. Zhang, Z. Zhang, "Impact behaviour of polypropylene filled with multi-walled carbon nanotubes," European polymer journal, vol. 43, no. 8, pp. 3197-3207, 2007.

[4] T. K. Tsotsis, "Continuous, carbon-nanotube-reinforced polymer precursors and carbon fibers," U.S. Patent No. 8, 187,700, 2012.

[5] F. T. Fisher, R. D. Bradshaw, and L. C. Brinson, "Fiber waviness in nanotube-reinforced polymer composites-I: Modulus predictions using effective nanotube properties," Composites Science and Technology, vol. 63, no. 11, pp. 1689-1703, 2003.

[6] K. Yanase, S. Moriyama, and J. W. Ju, "Effects of CNT waviness on the effective elastic responses of CNTreinforced polymer composites," Acta Mechanica, vol. 224, no. 7, pp. 1351-1364, 2013.

[7] J. Karger-Kocsis, H. Mahmood, and A. Pegoretti, "Recent advances in fiber/matrix interphase engineering for polymer composites," Progress in Materials Science, vol. 73, pp. 1-43, 2015.

[8] Y. J. Liu, nd X. L. Chen, "Evaluations of the effective material properties of carbon nanotube-based composites using a nanoscale representative volume element," Mechanics of materials, vol. 35, no. 1, pp. 69-81, 2003. 
[9] C. Li, and T.-W. Chou, "A structural mechanics approach for the analysis of carbon nanotubes," International Journal of Solids and Structures, vol. 40, no. 10, pp. 2487-2499, 2003.

[10] Y. Han, and J. Elliott, "Molecular dynamics simulations of the elastic properties of polymer/carbon nanotube composites," Computational Materials Science, vol. 39, no. 2, pp. 315-323, 2007.

[11] G. D. Seidel, and D. C. Lagoudas, "Micromechanical analysis of the effective elastic properties of carbon nanotube reinforced composites," Mechanics of Materials, vol. 38, no. 8, pp. 884-907, 2006.

[12] T. Mori, and K. Tanaka, "Average stress in matrix and average elastic energy of materials with misfitting inclusions," Acta metallurgica, vol. 21, no. 5, pp. 571-574, 1973.

[13] J. D. Eshelby, "The determination of the elastic field of an ellipsoidal inclusion, and related problems," Proceedings of the Royal Society of London A: Mathematical, Physical and Engineering Sciences., vol. 241, no. 1226, 1957. 